\title{
Analysis of Masticatory Muscle Tendon-aponeurosis Hyperplasia by Using Next-generation Sequencing
}

\author{
MEGUMI YUMOTO ${ }^{1}$, YOSUKE MIZUNO ${ }^{2}$, YUTA ISOZAKI ${ }^{1}$, \\ KO ITO ${ }^{1}$, TETSUYA YODA ${ }^{3}$ and TSUYOSHI SATO ${ }^{1}$ \\ ${ }^{1}$ Department of Oral and Maxillofacial Surgery, Saitama Medical University, Saitama, Japan; \\ ${ }^{2}$ Division of Analytical Science, Biomedical Research Center Hidaka Branch, \\ Saitama Medical University, Saitama, Japan; \\ ${ }^{3}$ Department of Maxillofacial Surgery, Graduate School of Medical and Dental Sciences, \\ Tokyo Medical and Dental University, Tokyo, Japan
}

\begin{abstract}
Background/Aim: Masticatory muscle tendonaponeurosis hyperplasia (MMTAH) is a disease associated with a mouth opening limitation. Here, we conducted a bioinformatics analysis to examine gene expression patterns in patients with MMTAH in comparison to those with facial deformity (FD). Materials and Methods: Seven MMTAH patients and three FD patients were recruited. We conducted RNA sequencing analysis, quantitative reverse transcription polymerase chain reaction and immunoblot analysis. Results: Of the identified 19,767 mapped read tags that showed clear differential expression, 2,471 genes were significantly up-regulated and 2,849 genes were significantly down-regulated in patients with MMTAH compared to those in patients with FD. Among the up-regulated genes, ten genes were significantly increased. The distribution of upregulated and down-regulated genes at different ages tended to be similar. Moreover, the protein levels of Ankyrin Repeat Domain 2, Troponin T1 and myosin heavy chain 7, which are associated with slow twitch fibers and mechanical loading, were strongly expressed in patients with MMTAH compared to those in patients with FD. Conclusion: The gene expression pattern in MMTAH patients was similar regardless of age. As the transition of fast-to-slow twitch in the skeletal muscle is induced by mechanical loading, and
\end{abstract}

This article is freely accessible online.

Correspondence to: Tsuyoshi Sato, Department of Oral and Maxillofacial Surgery, Saitama Medical University, 38 Morohongou, Moroyama-machi, Iruma-gun, Saitama, 350-0495, Japan. E-mail: tsato@saitama-med.ac.jp

Key Words: Masticatory muscle tendon-aponeurosis hyperplasia, next-generation sequencing, gene expression pattern, mechanical loading, slow twitch fiber. up-regulation of slow twitch molecules was observed in MMTAH patients, mechanical loading is suggested to be implicated in MMTAH.

Masticatory muscle tendon-aponeurosis hyperplasia (MMTAH) is a disease that causes limited mouth opening due to the hyperplasia of tendons and aponeuroses, which lead to a disturbance in muscle extensibility (1). The diagnosis of MMTAH depends on a palpable dense band at the anterior border of the masseter muscle upon maximal mouth opening. A secondary diagnostic feature is a square mandible configuration, which exhibits bilateral overhanging mandibular angles. Although Japanese clinicians first reported this disease in 2000, MMTAH was defined as a disease 8 years later. There have been few published reports on MMTAH outside of Japan (2).

On histological observation, MMTAH exhibits normal tendons and aponeuroses (1). However, we previously found that calcification was detected in the temporal tendon in MMTAH (3). Our previous study using proteome analysis revealed that beta-crystallin A4, which is increased by tensile force in tenocytes, is up-regulated in patients with MMTAH when compared to patients with facial deformity (FD) (4). Moreover, we showed that tendons in patients with MMTAH abundantly express decorin protein, which was induced by cyclic stretch via yes-associated protein signaling (5). The progression of this disease advances slowly from adolescence. Our previous investigation demonstrated that approximately $2 \%$ of 1,000 individuals in the domestic elementary and junior high school students are suspected of MMTAH (6). Therefore, we hypothesized that some genetic factors are involved in this disease.

RNA sequencing is a novel high-throughput sequencing method that uses deep-sequencing technology (7). This method has allowed many advances in the characterization and quantification of transcriptomes. However, few studies 
Table I. Basic characteristics of patients.

\begin{tabular}{lclcc}
\hline Diagnosis & No & Gender & Age & Parafunction \\
\hline MMATH & M1 & Female & 43 & None \\
& M2 & Female & 45 & None \\
& M3 & Female & 68 & None \\
& M4 & Female & 45 & None \\
& M5 & Female & 45 & None \\
& M6 & Female & 39 & None \\
FD & M7 & Female & 39 & Clenching \\
& F1 & Female & 46 & None \\
& F2 & Female & 26 & None \\
& F3 & Female & 19 & None \\
\hline
\end{tabular}

MMTAH: Masticatory muscle tendon-aponeurosis hyperplasia; FD: facial deformity.

have analyzed human tendons by RNA sequencing $(8,9)$. Herein, we explored differential gene expression in MMTAH by using RNA sequencing analysis.

\section{Materials and Methods}

Patients. Patients who were diagnosed with MMTAH or FD and underwent surgical treatment were included in this study (Table I). The patients with MMTAH had limited mouth opening within 35 $\mathrm{mm}$, no limitation of lateral or anterior mandibular movement, and a palpable overhanging master muscle. The patients with FD had no limitation concerning mouth opening. None of the patients had any other underlying disease, and were treated and followed up at our hospital. The present study was conducted according to the Declaration of Helsinki and also approved by the Institutional Review Board (number: 17027.07).

Sample preparation. Each tendon tissue, from which muscle tissue had been completely removed, was homogenized using Cell Destroyer (Biomedical Science, Tokyo, Japan).

Antibodies. We used anti-mouse MYH7 monoclonal antibody (MABT838; Merck Millipore, Danvers, MA, USA), anti-rabbit ANKRD2 polyclonal antibody (11821-1-AP; Proteintech, Rosemont, IL, USA), anti-rabbit TNNT1 polyclonal antibody (GTX109585; GeneTex, Irvine, CA, USA), and anti-rabbit alphatubulin polyclonal antibody (MBL, Nagoya, Japan) as primary antibodies. Anti-rabbit IgG horseradish peroxidase-linked antibody (Cell Signaling Technology, Danvers, MA, USA) and anti-mouse IgG horseradish peroxidase-linked antibody (Cell Signaling) were used as secondary antibodies.

RNA isolation. RNA was extracted using the miRNeasy Mini Kit (Qiagen, CA, USA). We checked the purity of the RNA by using a Nanodrop 2000 (Thermo Fisher Scientific, Waltham, MA, USA). We measured the RNA concentration by using the Qubit RNA Assay Kit (Life Technologies, Carlsbad, CA, USA). The integrity of the RNA was assessed by using the RNA Nano 6000 Assay Kit with the Agilent Bioanalyzer 2100 system (Agilent Technologies, Santa Clara, CA, USA).
Table II. Gene name and assay ID list.

\begin{tabular}{lc}
\hline Gene name & Assay ID \\
\hline ANKRD2 & Hs00220469_m1 \\
CA3 & Hs00193123_m1 \\
TNNT1 & Hs00162848_m1 \\
MYH7 & Hs01110632_m1 \\
FBXO40 & Hs00212488_m1 \\
MKX & Hs00543190_m1 \\
MYF5 & Hs00929416_g1 \\
MYF6 & Hs00231165_m1 \\
MYOD1 & Hs00159528_m1 \\
SMYD1 & Hs00400855_m1 \\
GAPDH & Hs02786624_g1 \\
\hline
\end{tabular}

Library preparation for RNA sequencing. For the preferential selection of cDNA fragments of 150 to $200 \mathrm{bp}$ in length, the library fragments were purified with the AMPure XP system (Beckman Coulter, MA, USA). PCR was performed with Phusion HighFidelity DNA polymerase. Finally, the products were purified by the AMPure XP system, and the library quality was assessed on the Agilent Bioanalyzer 2100 system.

Clustering and sequencing. The clustering of the index-coded samples was performed on a cBot Cluster Generation System using a HiSeq PE Cluster Kit cBot-HS (Illumina) according to the manufacturer's instructions. After cluster generation, the library preparations were sequenced on an Illumina Hiseq platform, and 150 paired-end reads were generated.

Mapping to the reference genome. The reference genome and gene model annotation files were downloaded directly from GenomeWeb (10). The index of the reference genome was built using Bowtie v2.0.6, and paired-end clean reads were aligned to the reference genome using TopHat v2.0.9.

Transcriptome assembly. The mapped reads of each sample were assembled by both Scripture (beta2) and Cufflinks (v2.1.1) in a reference-based approach $(11,12)$. Cufflinks uses a probabilistic model to simultaneously assemble and quantify the expression level of a minimal set of isoforms (13).

Coding potential analysis. We used Coding-Non-Coding-Index (CNCI) (v2) profiles adjoining nucleotide triplets to effectively distinguish protein-coding and non-coding sequences independent of known annotations (14). We used CNCI with the default parameters. We also used the Coding Potential Calculator (CPC) (0.9-r2), which mainly assesses the extent and quality of the open reading frames in a transcript, and searches for sequences in databases of known protein sequences to clarify the coding and noncoding transcripts (15). We used the NCBI eukaryotic protein database and set the e-value to ' $1 \mathrm{e}-10$ ' in our analysis.

Quantification of gene expression levels. Gene fragments per kilobase of exon per million fragments mapped (FPKMs) were computed by summing the FPKMs of transcripts in each gene 
A

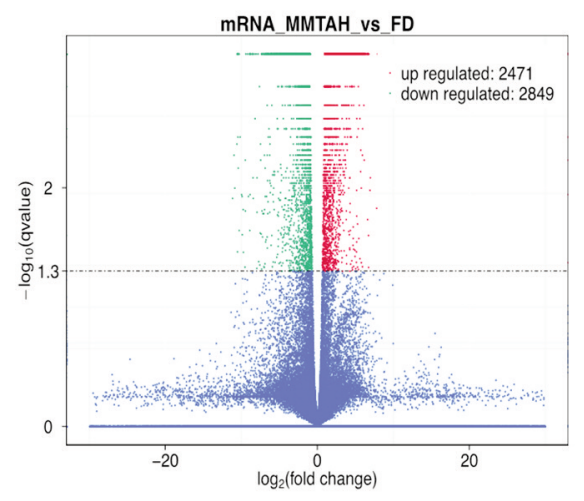

B

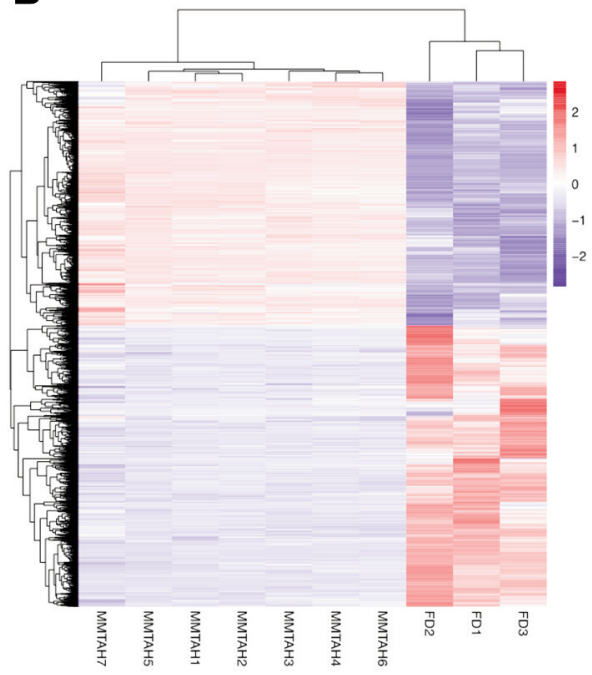

Figure 1. Expression profile obtained from RNA sequencing. (A) Volcano plot representation of the differential expression analysis of genes in patients with MMTAH in comparison to the data of the FD patients. Red and green points indicate the genes that were significantly up-regulated or down-regulated, respectively, in MMTAH samples when compared to FD samples. The x-axis shows the log2 fold changes in expression, and the $y$-axis shows the -log10 qvalue of a gene being differentially expressed. (B) Heat map representation with color-coded expression levels of the differentially expressed genes. Hierarchical clustering based on FPKMs, where log $10($ FPKM+1) is used for clustering. Red and blue colors indicate the genes that were significantly up-regulated or down-regulated, respectively, in the patients with MMTAH when compared to the patients with FD. MMTAH: Masticatory muscle tendon-aponeurosis hyperplasia; FD: facial deformity.

group. FPKMs were calculated based on the length of the fragments and read counts mapped to the fragment.

Differential expression analysis. For biological replicates, transcripts or genes with an adjusted $p<0.05$ were assigned as differentially expressed. For non-biological replicates, an adjusted $p<0.05$ and an absolute value of $\log 2$ (fold change) $<1$ were set as the thresholds for significant differential expression.

Gene ontology $(G O)$ enrichment analysis. GO enrichment analysis of the differentially expressed genes was performed using the GOseq R package, in which gene length bias was corrected. GO terms with a corrected $p$ value of less than 0.05 were considered to be significantly enriched in the differentially expressed genes.

Quantitative reverse transcription polymerase chain reaction ( $q R T$ $P C R$ ). The cDNA was synthesized by reverse-transcribing $0.5 \mu \mathrm{g}$ of RNA using the ReverTra Ace qPCR RT kit (Toyobo, Osaka, Japan). qPCR was carried out with a $20-\mu l$ reaction volume containing cDNA, the THUNDERBIRD Probe qPCR Mix (Toyobo), and TaqMan Gene Expression Assays (Thermo Fisher Scientific). We performed reactions at $95^{\circ} \mathrm{C}$ for $1 \mathrm{~min}$, followed by 40 cycles of $95^{\circ} \mathrm{C}$ for $15 \mathrm{~s}$, and $60^{\circ} \mathrm{C}$ for $30 \mathrm{~s}$ with Mx3000P STRATEGENE (Agilent Technologies). The primer sets used in this study are listed in Table II.

Immunoblot analysis. Tissue specimens that had been kept frozen in liquid nitrogen were cut into small pieces with scissors, and then lysed with $0.1 \%$ Triton X-100 containing ProteoGuard ${ }^{\text {TM }}$ EDTAFree Protease Inhibitor Cocktail (Takara, Shiga, Japan). Equal amounts of proteins were separated by sodium dodecyl sulfatepolyacrylamide gel electrophoresis. Subsequently, they were electro-blotted onto Immobilon PVDF membranes (Merck Millipore) at $100 \mathrm{~V}$ for $120 \mathrm{~min}$ using a Tank system (Cleaver Scientific, Warwickshire, UK). After blocking the non-specific binding by soaking the filters in $5 \%$ skim milk, we detected the required proteins with their respective antibodies. The membrane was developed using Clarity Western ECL Substrate (Bio-Rad, CA, USA). The bands were visualized by using a Chemi-Doc XRS system (Bio-Rad).

Statistical analysis. Comparisons between two groups were performed using the Student's $t$-test $\left({ }^{*} p<0.05 ; *^{* * *} p<0.01\right)$. All values represent the mean \pm S.E.M.

\section{Results}

To determine which genes were up-regulated in MMTAH in comparison to FD, RNA sequencing was performed on the RNA samples obtained from the temporal tendons. The expression profiles of 19,767 genes were collected from the RNA sequencing experiment. Among these genes, 2,471 genes were significantly up-regulated and 2,849 genes were significantly down-regulated (Figure 1A). A heat map revealed that there were specific differences within the transcriptome data, representing the known biological differences that exist between MMTAH and FD (Figure 1B). Moreover, the distribution of up-regulated genes and down- 
Table III. Top ten upregulated genes in MMTAH.

\begin{tabular}{|c|c|c|c|c|}
\hline Gene name & MMATH FPKM & FD FPKM & Fold change & $p$-Value \\
\hline Ankyrin repeat domain 2 (ANKRD2) & 306,801 & 1,12281 & 8,09405 & $p<0.001$ \\
\hline Carbonic anhydrase 3 (CA3) & 1030,01 & 4,58877 & 7,81033 & $p=0.0015$ \\
\hline Troponin T 1 (TNNT1) & 66,5557 & 0,528153 & 6,97746 & $p<0.001$ \\
\hline Myosin heavy chain 7 (MYH7) & 6227,8 & 58,0374 & 6,7456 & $p<0.001$ \\
\hline F-box protein 40 (FBXO40) & 92,9665 & 0,936542 & 6,63322 & $p<0.001$ \\
\hline SET and MYND domain containing 1 (SMYD1) & 62,3653 & 1,31484 & 5,56778 & $p<0.001$ \\
\hline Mohawk (MKX) & 5,0284 & 0,142784 & 5,13819 & $p<0.001$ \\
\hline Myogenic factor 6 (MYF6) & 89,4264 & 2,90258 & 4,94529 & $p<0.001$ \\
\hline Myogenic factor 5 (MYF5) & 1,75278 & 0,165454 & 3,40514 & $p=0.001$ \\
\hline Myoblast determination 1 (MYOD1) & 2,48773 & 0,257211 & 3,2738 & $p<0.001$ \\
\hline
\end{tabular}

MMTAH: Masticatory muscle tendon-aponeurosis hyperplasia; FD: facial deformity; FPKM: fragments per kilo-base of exon per million fragments mapped.

regulated genes at different ages tended to be similar, suggesting that patients with MMTAH show little change in the expression levels of these genes at different ages.

Among the GO terms related to biological processes, terms related to 'muscle' were highly enriched among the differentially expressed genes. We then focused on genes related to development and the differentiation of muscle and tendon cells. Among the GO terms including 'muscle cell development' and 'muscle cell differentiation', the following top 10 genes were up-regulated in the MMTAH samples when compared to the FD samples: ankyrin repeat domain 2 (ANKRD2), carbonic anhydrase $3(C A 3)$, troponin $\mathrm{T} 1$ (TNNT1), myosin heavy chain 7 (MYH7), F-box protein 40 (FBXO40), mohawk $(M K X)$, myogenic Factor 5 (MYF5), myogenic factor 6 (MYF6), myoblast determination protein 1 (MYOD1), and SET and MYND domain containing 1 (SMYD1) (Table III). We also analyzed the expression levels of these genes by qPCR, and found that these genes were significantly expressed in the MMTAH samples, similar to the RNA sequencing results (Figure 2).

It has been observed clinically that the tendon and muscle volume in MMTAH patients is increased, indicating that excessive mechanical loading is involved in the onset or development of MMTAH. Among the top 10 genes, we focused on molecules that are associated with slow twitch fibers, because mechanical loading induces fast-to-slow twitch transitions in the skeletal muscle (16). We found three molecules, i.e., ANKRD2, TNNT1, and MYH7, that are not only specifically expressed on slow twitch fibers, but are also involved in mechanical loading (17-19). We thus examined whether these molecules showed differential expression at the protein level in patients with FD and MMTAH. As expected, these proteins were highly expressed in patients with MMTAH when compared to those in patients with FD (Figure 3).

\section{Discussion}

The volume of the human tendon tissue increases only slightly after 20 years of age, and the synthesis of collagen is decreased due to aging $(20,21)$. Our results showed that the gene expression pattern in MMTAH patients did not differ with age. We speculate that the tendon in patients with MMTAH may not be normal.

In this study, a next-generation sequencing analysis revealed that the levels of more than 4,000 molecules were increased or decreased in the tendon of patients with MMTAH when compared to that of patients with FD. We focused on the up-regulated genes, because they may serve as specific diagnostic markers in the serum of patients with MMTAH. We identified several up-regulated genes that were associated with the differentiation of muscle and tendon in the MMTAH samples.

Mkx is a transcription factor expressed in various tendon tissues that controls tendon differentiation by regulating type I collagen production in tenocytes. It is also implicated in the response to mechanical stimuli (22). Mechanical loading, such as oral parafunction and aberrant habits, may cause tensile overloading on bilateral masticatory muscles. We have previously shown that the tendons of patients with MMTAH were subjected to cyclic stretch conditions (6). In the present study, the Mkx mRNA level was significantly increased in the tendons of patients with MMTAH, suggesting that Mkx up-regulation was due to oral parafunction and aberrant habits in MMTAH.

Myf5 and MyoD are essential regulators that determine the muscle progenitors at all anatomical sites of muscle formation within the developing vertebrate body. Myf5 controls the early process of progenitor specification, including cell proliferation and local cell migration, to establish progenitor stem cell pools at the sites of 

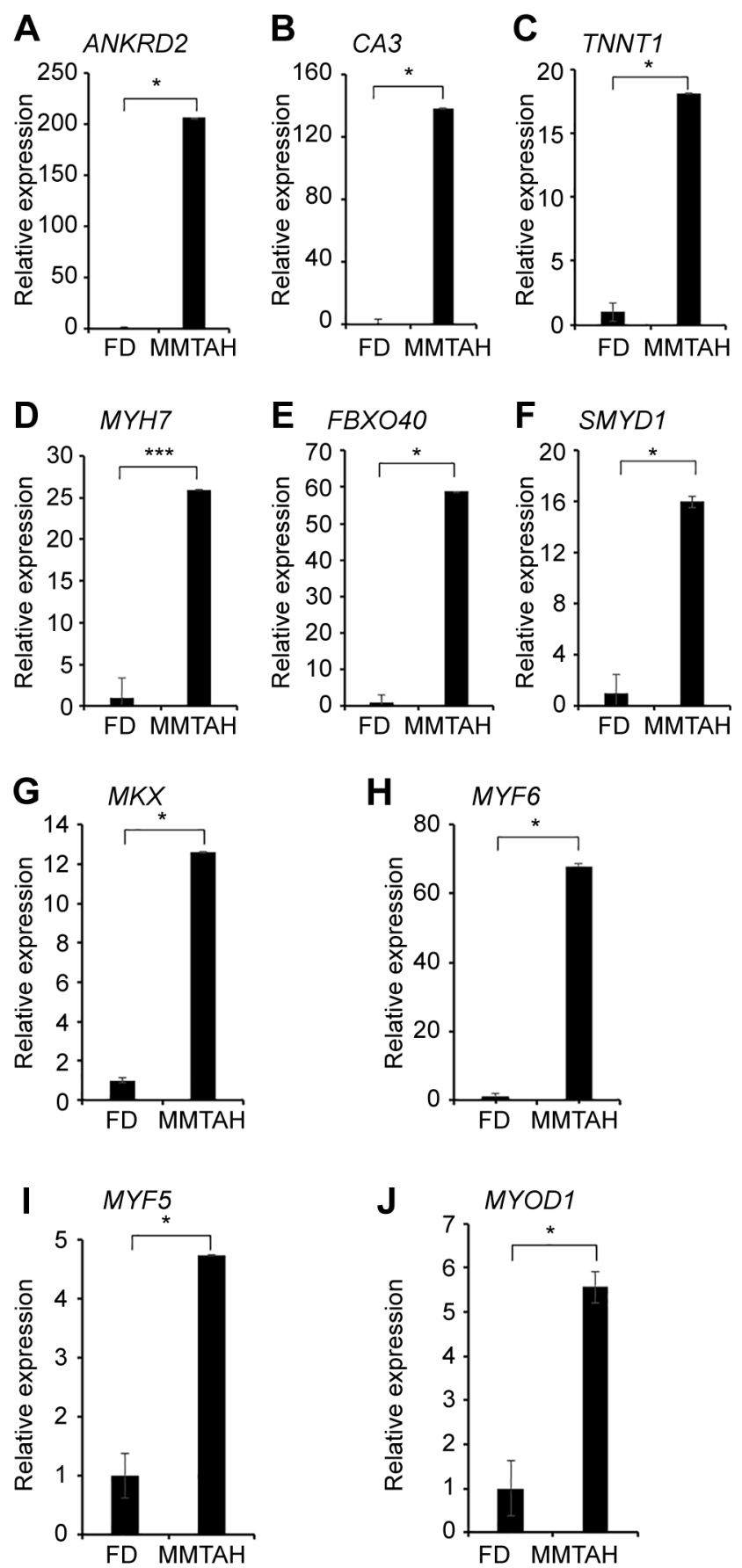

Figure 2. The top ten up-regulated myogenic and tenogenic genes in the MMTAH samples when compared to the FD samples. The gene expression levels of $(A)$ ankyrin repeat domain 2 (ANKRD2), (B) carbonic anhydrase 3 (CA3), (C) troponin T1 (TNNT1), (D) myosin heavy chain 7 (MYH7), (E) F-box protein 40 (FBXO40), (F) SET and $M Y N D$ domain containing 1 (SMYD1), (G) mohawk (MKX), (H) myogenic factor 6 (MYF6), (I) myogenic Factor 5 (MYF5), and (J) myoblast determination protein 1 (MYOD1) in the MMTAH or FD samples were estimated by $q R T-P C R .{ }^{*} p<0.05$. ${ }^{* * *} p<0.01$. Data are expressed as means \pm standard error of the mean. MMTAH: Masticatory muscle tendon-aponeurosis hyperplasia; FD: facial deformity.
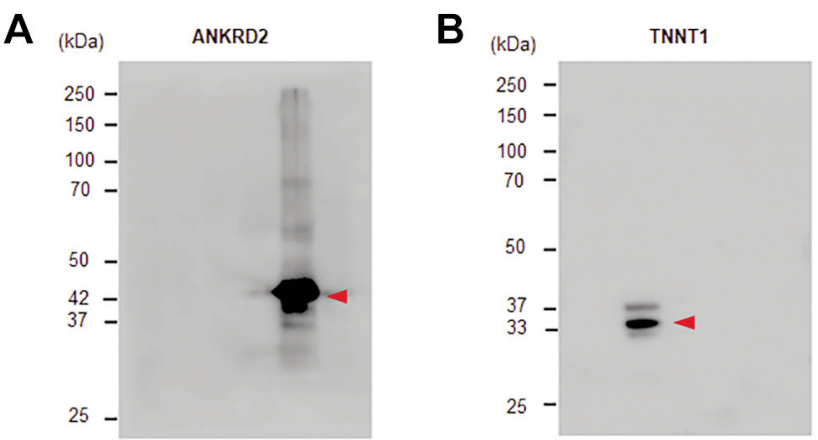

FD MMTAH
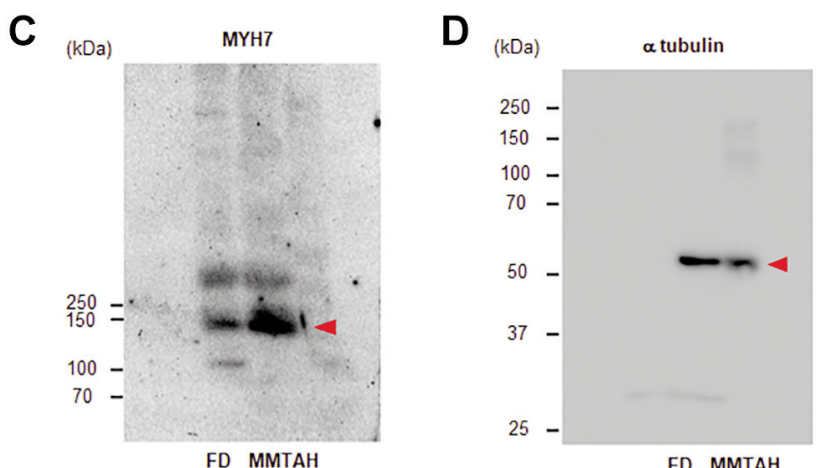

FD MMTAH

Figure 3. Increased protein levels of slow twitch molecules in patients with MMTAH. The protein levels of (A) ANKRD2, (B) TNNT1, (C) $M Y H 7$, and (D) a-tubulin, which was used as an internal control, in patients with MMTAH and FD patients were estimated by immunoblot analyses. MMTAH: Masticatory muscle tendon-aponeurosis hyperplasia; FD: facial deformity.

myogenesis, whereas MyoD functions in the initiation of differentiation and contractile protein gene expression (23, 24). Both Myf5 and MyoD regulate the myogenic lineage, as evidenced by the finding that double mutant mice do not form skeletal muscle as a result of the absence of precursor myoblasts, and they also play distinctive roles in cranial myogenesis (25-27). These findings indicate that the upregulation of Myf5 and MyoD may reflect the muscle hypertrophy in MMTAH.

In cases with slight oral parafunction, the patients are sometimes not aware of the symptom. Among the patients in the present study, no definitive oral parafunction was seen, except in patient M7. The heat map result of patient M7 differed from those of the other patients (Figure 1B), suggesting that oral parafunction affects the gene expression pattern. Since the transition of fast-to-slow twitch in the skeletal muscle is induced by mechanical loading, the upregulation of slow twitch molecules was expected in the tendons of the patients with MMTAH (16). Indeed, we found that slow twitch molecules, including ANKRD2, TNNT1, 
and MYH7, were highly expressed in the tendon tissues of patients with MMTAH (Figure 2 and Figure 3) (17-19). In contrast, the expression levels of fast twitch molecules, including MYH1m, MYH2, and MYH4, were not altered (28). These results suggested that mechanical loading is implicated in the pathological conditions of MMTAH.

This study has several limitations. Firstly, to determine the effect of environmental factors on gene expression, we need to examine age-matched patients with or without the environmental factors. Secondly, although the tendons of FD patients are normal, patients with FD are not normal subjects. However, we were unable to select able-bodied people for the control group due to ethical restrictions. Thirdly, we did not completely remove all muscle tissues at the micro-level. Microdissection would be useful for isolating tendon tissues. Finally, it remains unclear whether the up-regulation of the molecules that we identified was the result of environmental factors or the cause of the disease. To solve this problem, the data of chronological gene expression patterns from childhood and monozygotic gene expression patterns are required.

In summary, we obtained a glimpse of the global gene expression patterns in MMTAH by RNA sequencing. Further experiments are needed to determine whether these genes and molecules can be applied as diagnostic markers and/or therapeutic targets for MMTAH.

\section{Conflicts of Interest}

All Authors declare that they have no conflicts of interest in relation to this study.

\section{Authors' Contributions}

MY and YI conducted all the experiments, collected the data and drafted the manuscript. MY, TS and YM analyzed the data. KI and TY critically revised the manuscript. TS designed the study and drafted the manuscript. All Authors gave final approval of the manuscript and agreed to be accountable for all aspects of the work.

\section{Acknowledgements}

This work was supported by JSPS KAKENHI Grant Number JP21H03137 to Tetsuya Yoda.

\section{References}

1 Sato T and Yoda T: Masticatory muscle tendon-aponeurosis hyperplasia: A new clinical entity of limited mouth opening. Jpn Dent Sci Rev 52(2): 41-48, 2016. PMID: 28408955. DOI: 10.1016/j.jdsr.2015.11.001

2 Worni A, Mericske-Stern R, Iizuka T and Büttner M: [Limited mouth opening - what now?]. Swiss Dent J 124(9): 935-944, 2014. PMID: 25253540 .
3 Sato T, Hori N, Nakamoto N, Akita M and Yoda T: Masticatory muscle tendon-aponeurosis hyperplasia exhibits heterotopic calcification in tendons. Oral Dis 20(4): 404-408, 2014. PMID: 23750917. DOI: 10.1111 /odi.12140

4 Hayashi N, Sato T, Yumoto M, Kokabu S, Fukushima Y, Kawata Y, Kajihara T, Mizuno Y, Mizuno Y, Kawakami T, Kirita T, Hayata T, Noda M and Yoda T: Cyclic stretch induces decorin expression via yes-associated protein in tenocytes: a possible mechanism for hyperplasia in masticatory muscle tendonaponeurosis hyperplasia. J Oral Maxillofac Surg Med Pathol 31(3): 175-179, 2019. DOI: 10.1016/j.ajoms.2018.12.012

5 Hayashi N, Sato T, Kokabu S, Usui M, Yumoto M, Ikami E, Sakamoto Y, Nifuji A, Hayata T, Noda M and Yoda T: Possible association of oestrogen and Cryba4 with masticatory muscle tendon-aponeurosis hyperplasia. Oral Dis 25(1): 274-281, 2019. PMID: 29683234. DOI: 10.1111/odi.12876

6 Hayashi N, Yoda T, Tomoda T, Yumoto M, Kitamura T, Okubo M, Iwasaki Y, Enoki Y and Sato T: Longitudinal epidemiological survey on suspected masticatory muscle tendon-aponeurosis hyperplasia in adolescents. J Jpn Soc TMJ 30: 187-194, 2018. DOI: $10.11246 /$ gakukansetsu.30.187

7 Wang Z, Gerstein M and Snyder M: RNA-Seq: a revolutionary tool for transcriptomics. Nat Rev Genet 10(1): 57-63, 2009. PMID: 19015660 . DOI: $10.1038 / \mathrm{nrg} 2484$

8 Peffers MJ, Fang Y, Cheung K, Wei TK, Clegg PD and Birch HL: Transcriptome analysis of ageing in uninjured human Achilles tendon. Arthritis Res Ther 17: 33, 2015. PMID: 25888722. DOI: 10.1186/s13075-015-0544-2

9 Pease LI, Clegg PD, Proctor CJ, Shanley DJ, Cockell SJ and Peffers MJ: Cross platform analysis of transcriptomic data identifies ageing has distinct and opposite effects on tendon in males and females. Sci Rep 7(1): 14443, 2017. PMID: 29089527. DOI: 10.1038/s41598-017-14650-z

10 GenomeWeb. Available at: https://www.genomeweb.com [Last accessed on January 24, 2022]

11 Guttman M, Garber M, Levin JZ, Donaghey J, Robinson J, Adiconis X, Fan L, Koziol MJ, Gnirke A, Nusbaum C, Rinn JL, Lander ES and Regev A: Ab initio reconstruction of cell typespecific transcriptomes in mouse reveals the conserved multiexonic structure of lincRNAs. Nat Biotechnol 28(5): 503-510, 2010. PMID: 20436462. DOI: $10.1038 /$ nbt.1633

12 Trapnell C, Williams BA, Pertea G, Mortazavi A, Kwan G, van Baren MJ, Salzberg SL, Wold BJ and Pachter L: Transcript assembly and quantification by RNA-Seq reveals unannotated transcripts and isoform switching during cell differentiation. Nat Biotechnol 28(5): 511-515, 2010. PMID: 20436464. DOI: $10.1038 /$ nbt.1621

13 Cabili MN, Trapnell C, Goff L, Koziol M, Tazon-Vega B, Regev A and Rinn JL: Integrative annotation of human large intergenic noncoding RNAs reveals global properties and specific subclasses. Genes Dev 25(18): 1915-1927, 2011. PMID: 21890647. DOI: $10.1101 / \operatorname{gad} .17446611$

14 Sun L, Luo H, Bu D, Zhao G, Yu K, Zhang C, Liu Y, Chen R and Zhao Y: Utilizing sequence intrinsic composition to classify protein-coding and long non-coding transcripts. Nucleic Acids Res 41(17): e166, 2013. PMID: 23892401. DOI: 10.1093/nar/ gkt646

15 Kong L, Zhang Y, Ye ZQ, Liu XQ, Zhao SQ, Wei L and Gao G: CPC: assess the protein-coding potential of transcripts using sequence features and support vector machine. Nucleic Acids 
Res 35(Web Server issue): W345-W349, 2007. PMID: 17631615. DOI: $10.1093 / \mathrm{nar} / \mathrm{gkm} 391$

16 Pette D and Staron RS: Myosin isoforms, muscle fiber types, and transitions. Microsc Res Tech 50(6): 500-509, 2000. PMID: 10998639. DOI: 10.1002/1097-0029(20000915)50:6<500::AIDJEMT7>3.0.CO;2-7

17 Mohamed JS, Lopez MA, Cox GA and Boriek AM: Anisotropic regulation of Ankrd2 gene expression in skeletal muscle by mechanical stretch. FASEB J 24(9): 3330-3340, 2010. PMID: 20442316. DOI: 10.1096/fj.10-158386

18 Heher P, Maleiner B, Prüller J, Teuschl AH, Kollmitzer J, Monforte X, Wolbank S, Redl H, Rünzler D and Fuchs C: A novel bioreactor for the generation of highly aligned 3D skeletal muscle-like constructs through orientation of fibrin via application of static strain. Acta Biomater 24: 251-265, 2015. PMID: 26141153. DOI: 10.1016/j.actbio.2015.06.033

19 Bell ML, Buvoli $M$ and Leinwand LA: Uncoupling of expression of an intronic microRNA and its myosin host gene by exon skipping. Mol Cell Biol 30(8): 1937-1945, 2010. PMID: 20154144. DOI: 10.1128/MCB.01370-09

20 Józsa L, Kvist M, Bálint BJ, Reffy A, Järvinen M, Lehto M and Barzo M: The role of recreational sport activity in Achilles tendon rupture. A clinical, pathoanatomical, and sociological study of 292 cases. Am J Sports Med 17(3): 338-343, 1989. PMID: 2729483. DOI: 10.1177/036354658901700305

21 Kannus P: Structure of the tendon connective tissue. Scand J Med Sci Sports 10(6): 312-320, 2000. PMID: 11085557. DOI: 10.1034/j.1600-0838.2000.010006312.x

22 Kayama T, Mori M, Ito Y, Matsushima T, Nakamichi R, Suzuki H, Ichinose S, Saito M, Marumo K and Asahara H: Gtf2ird1dependent Mohawk expression regulates mechanosensing properties of the tendon. Mol Cell Biol 36(8): 1297-1309, 2016. PMID: 26884464. DOI: 10.1128/MCB.00950-15
23 Pownall ME, Gustafsson MK and Emerson CP Jr: Myogenic regulatory factors and the specification of muscle progenitors in vertebrate embryos. Annu Rev Cell Dev Biol 18: 747-783, 2002. PMID: 12142270. DOI: 10.1146/annurev.cellbio.18.012502. 105758

24 Bergstrom DA, Penn BH, Strand A, Perry RL, Rudnicki MA and Tapscott SJ: Promoter-specific regulation of MyoD binding and signal transduction cooperate to pattern gene expression. Mol Cell 9(3): 587-600, 2002. PMID: 11931766. DOI: 10.1016/ s1097-2765(02)00481-1

25 Rudnicki MA, Schnegelsberg PN, Stead RH, Braun T, Arnold $\mathrm{HH}$ and Jaenisch R: MyoD or Myf-5 is required for the formation of skeletal muscle. Cell 75(7): 1351-1359, 1993. PMID: 8269513. DOI: 10.1016/0092-8674(93)90621-v

26 Kaul A, Köster M, Neuhaus H and Braun T: Myf-5 revisited: loss of early myotome formation does not lead to a rib phenotype in homozygous Myf-5 mutant mice. Cell 102(1): 1719, 2000. PMID: 10929709. DOI: 10.1016/s0092-8674(00) 00006-4

27 Lin CY, Yung RF, Lee HC, Chen WT, Chen YH and Tsai HJ: Myogenic regulatory factors Myf5 and Myod function distinctly during craniofacial myogenesis of zebrafish. Dev Biol 299(2): 594-608, 2006. PMID: 17007832. DOI: 10.1016/j.ydbio. 2006.08 .042

28 Schiaffino S and Reggiani C: Fiber types in mammalian skeletal muscles. Physiol Rev 91(4): 1447-1531, 2011. PMID: 22013216. DOI: $10.1152 /$ physrev.00031.2010
Received January 4, 2022

Revised January 20, 2022 Accepted January 24, 2022 